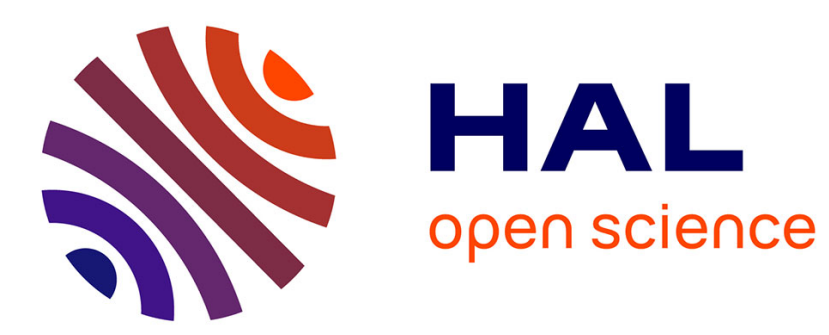

\title{
DUALITY AND GENERAL EQUILIBRIUM THEORY UNDER KNIGHTIAN UNCERTAINTY *
}

\author{
Patrick Beissner, Laurent Denis
}

\section{To cite this version:}

Patrick Beissner, Laurent Denis. DUALITY AND GENERAL EQUILIBRIUM THEORY UNDER KNIGHTIAN UNCERTAINTY *. SIAM Journal on Financial Mathematics, 2018, 10.1137/17M1120877 . hal-01585973

\section{HAL Id: hal-01585973 https://hal.science/hal-01585973}

Submitted on 12 Sep 2017

HAL is a multi-disciplinary open access archive for the deposit and dissemination of scientific research documents, whether they are published or not. The documents may come from teaching and research institutions in France or abroad, or from public or private research centers.
L'archive ouverte pluridisciplinaire HAL, est destinée au dépôt et à la diffusion de documents scientifiques de niveau recherche, publiés ou non, émanant des établissements d'enseignement et de recherche français ou étrangers, des laboratoires publics ou privés. 


\title{
DUALITY AND GENERAL EQUILIBRIUM THEORY UNDER KNIGHTIAN UNCERTAINTY*
}

\author{
PATRICK BEISSNER ${ }^{\dagger}$ AND LAURENT DENIS $\ddagger$
}

\begin{abstract}
Any dynamic or stochastic notion of a general equilibrium relies on the underlying commodity space. Under sole risk and without multiple-prior uncertainty the usual choice is a Lebesgue space from standard measure theory. In the case of volatility uncertainty it turns out that such a type of function space is no longer appropriate. For this reason we introduce and discuss a new natural commodity space, which can be constructed in three independent and equivalent ways. Each approach departs from one possible way to construct Lebesgue spaces.

Moreover, we give a complete representation of the resulting topological dual space. This extends the classic Riesz representation in a natural way. Elements therein are the candidates for a linear equilibrium price system. This representation result has direct implications for the microeconomic foundation of finance under Knightian uncertainty.
\end{abstract}

Key words. Asset pricing, general equilibrium under uncertainty, space of contingent claims, volatility uncertainty, dual space, mutually singular probability measures

AMS subject classifications. 91B51, 91G80

1. Introduction. The modern approach to neoclassic asset pricing and mathematical finance relies on the general equilibrium foundation in which risk, quantified by a single objective probability measure, is identified with uncertainty. By breaking such traditionally strong ties, the goal of the present paper is to analyze the basic link between absence of arbitrage and linear prices under volatility uncertainty in continuous time. Under multiple prior uncertainty, in the sense of [?], this usually strong link stops to work in the usual way. In order to understand this conceptually important issue we go back to the fundamentals and start from scratch.

From an empirical perspective, we consider a fairly general form of volatility uncertainty. However, we restrict our discussion to sets of priors $\mathscr{P}$ that are weakly compact. This covers any collection of stochastic volatility models that is bounded above by some Hölder continuous function. With regard to observed volatility patterns in financial markets, this is a rather weak assumption since any observed volatility (per annum) is contained in a bounded interval, say [1\%,200\%]. Note that this includes volatility patterns during the peak of the last financial crisis, see [?].

Under volatility uncertainty, the central arbitrage concept and the connection to competitive equilibria (with linear prices) goes against usual wisdom, see [?] for a detailed account of the usual connection. To understand the main differences, it is enlightening to consider related questions of pricing derivatives. In particular, volatility uncertainty creates an "intrinsically incomplete" market, see [?] for superhedging prices of standard European options. However, in the present framework it is also important to understand the pricing of volatility derivatives, see [?] for an overview of this rather new class of financial products. We show in a simple example that in a non-pathological Arrow-Debreu equilibrium one can construct a whole family of pos-

\footnotetext{
*Submitted to the editors August 30, 2017.

Funding: The work of L. Denis is supported by research project "PANORisk" - Région Pays de la Loire and project "CAESARS" (ANR-15-CE05-0024)

${ }^{\dagger}$ Research School of Economics - Australian National University, Canberra Australia. patrick.beissner@anu.edu.au,

${ }^{\ddagger}$ Laboratoire Manceau de Mathematiques - Universite du Maine, Le Mans France. ldenis@univlemans.fr
} 
itive (digital) volatility derivatives which are costless when priced by the equilibrium pricing measure.

From a theoretical perspective, it is important to recognize that the foundation of modern asset pricing has its origins within the conceptual building block of a general equilibrium theory à la Arrow-Debreu. Following this perspective, the present type of model uncertainty requires a new commodity space of all contingent claims within the model. As a conceptional point on the wishlist, the new commodity space of contingent claims (or random variables) should to some extent be a canonical extension of the classic Lebesgue space, see [?]. In finance models it is common to take the $L^{2}(P)$-space of square-integrable random variables under some given "objective" probability space $P$. When moving to a multiple-prior setting, one may hope to find a new space of random variables that shares many properties with the usual $L^{2}$-space. This is of particular importance when it comes to asset pricing. Price functionals are usually elements of the dual space which is determined by the (norm of the) primal space. An intuitive and explicit representation of any dual element plays a pivotal role in developing a theory of finance with a microeconomic foundation.

A natural generalization of Lebesgue spaces when moving to a set of probability measures $\mathscr{P}$ is achieved in Theorem 1. We depart from [?] and present three independent methods to construct a Lebesgue-type space and show that all these constructions result in the same Banach lattice of random variables, denoted by $L^{2}(\mathscr{P})$. With this robust generalization of a space of square integrable random variables, we turn to its topological dual and achieve a generalized version of the Riesz representation theorem in Theorem 2. It states that any linear and norm-continuous functional $\Pi$ on $L^{2}(\mathscr{P})$ can be represented as $\Pi(\cdot)=E^{P}[\psi \cdot]$ for some $P \in \mathscr{P}$ and some $\psi \in L^{2}(P)$. In macro finance applications $\psi$ is directly linked to the stochastic discount factor and play a pivotal role in most asset pricing models.

This established commodity-price duality under Knightian uncertainty allows then to analyze a related class of general equilibrium models under uncertainty. Here, the relation between an equilibrium price system and a (risk neutral) pricing measure requires a careful reconsideration. Under the present type of volatility uncertainty, a set of possibly mutually singular probability measures $\mathscr{P}$ emerges. Usually, such probability measures live on disjoint domains. This has direct implications for the meaning of an "equivalent" pricing measure. For instance, the first introduction of an equivalent-martingale measure appeared in [?] and is deduced from a (singleagent) equilibrium notion of viability. In such a frictionless model of competition, the resulting price system (a functional on the space of all possible contingent claims) is usually assumed to be linear and positive. From our Theorem 2 on the representation of any linear and continuous functional we can then infer that such price systems are stuck to the domain of a particular measure $P \in \mathscr{P}$. This in turn opens the door to arbitrage opportunities outside the domain of the representing $P$.

Related Literature. First models of uncertain volatility for financial market models can be found in [?] and [?]. In the same vein, [?] initiated a stochastic calculus under uncertain volatility. Recently, [?, ?] introduces a dynamic single-agent equilibrium notion under uncertain volatility. Robust utility maximization is considered in [?]. [?] presents no-arbitrage considerations under volatility uncertainty.

The paper is organized as follows. In Section 2 we introduce the uncertainty model. Section 3 establishes the commodity space via three independent construction methods. Section 4 gives an explicit representation of the topological dual. With these rather mathematical preparations, we discuss in Section 5 the implication for Arrow- 
Debreu equilbira and present several examples. Section 6 discusses some inconsistencies with linear-price system and no arbitrage. In Section 7 we conclude. The Appendix A prepares the proofs of the main results. Appendix B presents the proofs.

2. Knightian Uncertainty about Volatility. The construction of priors requires some structure on the state space $\Omega$. Assume $\Omega=\left\{\omega \in C([0, T] ; \mathbb{R}): \omega_{0}=0\right\}$ to be the space of continuous sample paths starting at zero, equipped with the supnorm. More generally it suffices to assume that $\Omega$ is a polish space. The Borel $\sigma$-field of $\Omega$ is denoted by $\mathcal{F}=\mathcal{B}(\Omega)$. Let $\Delta(\Omega)$ be the set of all probability measures on $(\Omega, \mathcal{F})$.

2.1. Construction of Probability Measures. Under the Wiener measure $P_{0}$, the canonical process $B_{t}(\omega)=\omega_{t}$ becomes a Brownian motion. Denote by $\mathbb{F}=$ $\left(\mathcal{F}_{t}\right)_{t \in[0, T]}$, with $\mathcal{F}_{t}=\sigma\left(B_{s}, s \in[0, t]\right)$, the information flow of the state process. Ambiguous volatility is based upon martingale laws of the form $P^{\sigma}=P_{0} \circ\left(B^{\sigma}\right)^{-1}$, where $B_{t}^{\sigma}=\int_{0}^{t} \sigma_{s} \mathrm{~d} B_{s}$ is a Brownian martingale under $P_{0}$ with quadratic variation $\left\langle B^{\sigma}\right\rangle_{t}=\int_{0}^{t} \sigma_{s}^{2} \mathrm{~d} s$. Each adapted stochastic volatility process $\sigma: \Omega \times[0, T] \rightarrow \mathbb{R}$ generates the probability law $P^{\sigma}$ of $B^{\sigma}$ on $\Omega$. Assume that the uncertainty is generated by a convex and bounded set $\mathcal{D}=\left\{\sigma_{t} \in\left[\underline{\sigma}_{t}, \bar{\sigma}_{t}\right]\right\}$ of positive processes. The set of priors is weakly compact and given by $\mathscr{P}=\left\{P^{\sigma}: \sigma \in \mathcal{D}\right\}$.

The purpose of $P_{0}=P^{1}$ stems from the construction of the uncertainty model and is not a reference measure. $P_{0} \notin \mathscr{P}$ means that $1 \notin \mathcal{D}$. To guarantee weak compactness and convexity of $\mathscr{P}$, it suffices to assume Hölder continuity of the bounds $\underline{\sigma}, \bar{\sigma}:[0, T] \rightarrow \mathbb{R}$ for $\left(\sigma_{t}\right)$, see Proposition 5 in [?]. Probability measures in $\mathscr{P}$ happen to be mutually singular. Our approach also covers simultaneous drift and volatility uncertainty.

EXAMPLE 1. 1. We consider a set of probabilities $\mathscr{P}$, in which both volatility and drift of some state process are uncertain. Consider some finite constants $0<\underline{\sigma}<\bar{\sigma}$ and $\underline{\kappa}<\bar{\kappa}$. We denote by $\mathscr{P}(\underline{\sigma}, \bar{\sigma}, \underline{\kappa}, \bar{\kappa})$ the set of probability laws on $(\Omega, \mathcal{F})$ of all the continuous semimartingales $S$ which admit the decomposition $S_{t}=\int_{0}^{t} \sigma_{s} \mathrm{~d} B_{s}+\int_{0}^{t} \theta_{s} \mathrm{~d} s$, where $B$ is a Brownian motion, $\sigma$ is predictable and $\theta$ is adapted. These processes are defined on a filtered probability space with usual conditions and satisfy $\underline{\sigma} \leq \sigma_{t} \leq \bar{\sigma}$ and $\underline{\kappa} \leq \theta_{t} \leq \bar{\kappa}$ for all $t \leq T$, $P_{0}$-a.s.

2. As a special case of part 1., the resulting sublinear expectation corresponds to the $G$-expectation, see [?], if $\underline{\kappa}=\bar{\kappa}=0$

In view of Example 1, we then have by Proposition 5 in [?]: $\mathscr{P}(\underline{\sigma}, \bar{\sigma}, \underline{\kappa}, \bar{\kappa})$ is a convex and weakly compact set in $\Delta(\Omega)$.

3. The Space of Contingent Claims. This section discusses a possible substitute for the standard Lebesgue space, when multiple priors describe the uncertainty. Some important special cases are discussed in the examples of Section 2.

3.1. Three Constructions for one Function Space. We consider the case of an abstract uncertainty model that is given by a weakly compact set of (possibly) mutually singular probability measures $\mathscr{P}$. Each $P \in \mathscr{P}$ is defined on $(\Omega, \mathcal{F})$, where $\Omega$ is an arbitrary polish space and $\mathcal{F}=\mathcal{B}(\Omega)$. A natural question then points to the formulation of a canonical function space of random variables. In the following, we present three different methods of how to generalize the measure theoretic Lebesgue space $L^{p}(P):=L^{p}(\Omega, \mathcal{F}, P)$ with finite $p$-th moment $\|X\|_{P, p}=E^{P}\left[|X|^{p}\right]^{1 / p}$. 


\section{Method 1: Topological Completion}

To describe an $\mathcal{F}$-measurable claim $X: \Omega \rightarrow \mathbb{R}$ under $\mathscr{P}$, we focus on the sublinear expectation ${ }^{1} \mathbb{E}[X]=\max _{P \in \mathscr{P}} E^{P}[X]$ and the resulting generalized $L^{p}$ type norm $\|X\|_{\mathscr{P}, p}=\mathbb{E}\left[|X|^{p}\right]^{1 / p}$. The closure of $\mathcal{C}_{b}(\Omega)$, the space of continuous and bounded functions on $\Omega$, under $\|\cdot\|_{\mathscr{P}, p}$ is denoted by $\mathcal{L}^{p}(\mathscr{P})$. The space

$$
L^{p}(\mathscr{P})=\overline{\mathcal{C}_{b}(\Omega)}\|\cdot\| \mathscr{P}, p / \mathcal{N}
$$

denotes the quotient space of $\mathcal{L}^{p}(\mathscr{P})$ with respect to $\mathcal{N}$. Here, $\mathcal{N}:=\mathcal{N}_{\mathscr{P}}$ denotes the collection of all $\|\cdot\|_{\mathscr{P}}$-null elements; functions that are null $\mathscr{P}$-quasi surely. A property holds $\mathscr{P}$-quasi surely (q.s.) if it holds $P$-a.s. for every $P \in \mathscr{P}$. We do not distinguish between classes and their representatives. $X, X^{\prime} \in L^{p}(\mathscr{P})$ can be distinguished if there is a $P \in \mathscr{P}$ with $P\left(X \neq X^{\prime}\right)>0$. As shown in [?], $L^{p}(\mathscr{P})$ is a Banach space.

\section{Method 2: Representation via quasi-continuous functions}

Another approach departs from the perspective of Lusin's Theorem. Under minimal topological conditions on $\Omega$, Borel measurable functions are quasi-continuous. ${ }^{2}$ Departing from this classic result we base the second construction on the concept of quasi-continuity. We say that $X: \Omega \rightarrow \mathbb{R}$ has a q.c. version if there exists a quasicontinuous function $Y: \Omega \rightarrow \mathbb{R}$ with $X=Y$ q.s. In that case, we write $X \in \mathcal{Q C}$. A mapping $X: \Omega \rightarrow \mathbb{R}$ is said to be quasi-continuous (q.c.) if for all $\varepsilon>0$ there exists an open set $O$ with $\mathbb{C}_{\mathscr{P}}(O):=\sup _{P \in \mathscr{P}} P(O)<\varepsilon$ such that $\left.X\right|_{O^{c}}$ is continuous. Define

$$
L^{p}(\mathbb{E})=\left\{X \in L(\Omega):\|X\|_{\mathscr{P}, p}<\infty, X \in \mathcal{Q C}, \lim _{n \rightarrow \infty} \mathbb{E}\left[\mathbf{1}_{\left\{|X|^{p}>n\right\}}|X|\right]=0\right\},
$$

where $L(\Omega)$ denotes the space of equivalence classes (with respect to $\mathcal{N}$ from Method 1) of $\mathcal{F}$-measurable random variables. The third requirement within the representation of (2) is a uniform integrability condition under $\mathbb{E}$.

\section{Method 3: Choquet's Lebesgue Prolongation via Capacities}

An alternative (and less known) way to construct function spaces with a finite capacity norm was initiated by [?]. [?] presents a more detailed description, see also [?]. Let $\mathcal{H}$ be any vector lattice in $\mathcal{C}_{b}(\Omega)$, containing constants and generating the topology of $\Omega$. The prolongation is induced by a sublinear expectation $\mathbb{E}$ via $c_{\mathbb{E}}^{p}(\phi)=\mathbb{E}\left[|\phi|^{p}\right]^{\frac{1}{p}}$ for all $\phi \in \mathcal{H}$ in the following way:

- Set $c_{\mathbb{E}}^{p}(f)=\sup \left\{c_{\mathbb{E}}^{p}(\phi): 0 \leq \phi \leq f, \phi \in \mathcal{H}\right\}$ for any $f \geq 0$ and lower semicontinuous (lsc).

- For any $X \in L(\Omega)$ set $c_{\mathbb{E}}^{p}(X)=\inf \left\{c_{\mathbb{E}}^{p}(f): f \geq|X|, f l s c\right\}$.

We can now define the function space induced by the Lebesgue prolongation:

$$
L\left(c_{\mathbb{E}}^{p}\right)=\mathrm{CL}_{c_{\mathbb{E}}^{p}}(\mathcal{H})_{/ \mathcal{N}}
$$

where $\mathrm{CL}_{c_{\mathbb{R}}^{p}}$ denotes the closure operation with respect to the norm $c_{\mathbb{E}}^{p}$ in the set $\left\{g \in L(\Omega): c_{\mathbb{E}}^{p}(g)<\infty\right\}$. Similar to Method 1, we consider equivalence classes that are now induced by the polar sets $\mathcal{N}$ of $c_{\mathbb{E}}^{p}{ }^{3}$

\footnotetext{
${ }^{1} \mathrm{By}[?], \mathbb{E}$ is a sublinear expectation, i.e. monotonicity, positive homogeneity, a constant preserving property, sub-additivity.

${ }^{2}$ For a detailed formulation of Lusin's Theorem we refer to Appendix D in [?].

${ }^{3}$ It is important to note that we have in fact to set capacities. Indeed, for any $A \in \mathcal{B}(\Omega)$ we can put $\mathbb{C}(A):=c_{\mathbb{E}}^{1}\left(1_{A}\right)$. One can prove that in general $c_{\mathbb{E}}^{1}(A) \neq \mathbb{C}_{\mathscr{P}}(A)$. As shown in Appendix A.2, the equality holds for $A$ open or compact. From this, it follows that there is no ambiguity in the term "quasi-continuous", when connecting method 2 and 3.
} 
3.2. Invariance of the Constructions. It turns out that all the three approaches, which respectively constructs a function space under Knightian uncertainty $\mathscr{P}$, result in the same space. This insight is the content of the first main result.

Theorem 1. (Representation of the Primal Space) Let $\Omega$ be a polish space and $\mathscr{P}$ be a weakly compact set in $\Delta(\Omega)$. For any $p \in[1, \infty)$, the spaces in (1), (2) and (3) are Banach spaces and coincide, i.e.

$$
L^{p}(\mathbb{E})=L^{p}(\mathscr{P})=L\left(c_{\mathbb{E}}^{p}\right)
$$

To some extend, Theorem 1 is remarkable, as the three constructions of function spaces follow rather different ideas; classical completion of a test space in (1), Luisin's Theorem in (2) and the capacity-based two step procedure in (3). From this perspective, Theorem 1 serves as a formal justification that $L^{p}(\mathscr{P})$ is "robust" against different construction methods.

The order structure on $L^{p}(\mathscr{P})$ is then given by $C \geq C^{\prime}$ if $C \geq C^{\prime}$ q.s. ( $\mathscr{P}$-quasi surely) and makes the triplet $\left(L^{p}(\mathscr{P}),\|\cdot\|_{\mathscr{P}, p}, \geq\right)$ into an order continuous Banach lattice, see Proposition 1 in [?]. The induced positive cone is denoted by $L^{p}(\mathscr{P})_{+}$.

REMARK 1. 1. Theorem 1 heavily depends on the weak compactness of $\mathscr{P}$. As characterized in [?], this is equivalent to the regularity of the capacity $\mathbb{C}_{\mathscr{P}}$. We also note, that the first equality of Theorem 1 is shown in [?]. The second equality is crucial for Theorem 2.

2. Alternatively one could consider in Method 1 the completion of the larger space $B_{b}(\Omega)$, the collection of all bounded measurable random variables. As discussed in [?], the completion is strictly larger than $L^{p}(\mathscr{P})$ and can be represented similarly as in Method 2, by

$$
\left\{X \in L(\Omega):\|X\|_{\mathscr{P}, p}<\infty, \lim _{n \rightarrow \infty} \mathbb{E}\left[\mathbf{1}_{\left\{|X|^{p}>n\right\}}|X|\right]=0\right\} .
$$

3. The generality of the approach also allows to consider the function space of adapted stochastic processes. In that case, we depart from the polish space $\Omega=C_{0}([0, T]) \times[0, T]$. In Subsection 4.3, we then state the dual for the function space of progressively measurable processes.

4. The Topological Dual Space. We recall the basic relation of Lebesgue spaces $L^{p}$ and the representation of the topological dual, that is, the collection of all linear and continuous functionals $\Pi: L^{p} \rightarrow \mathbb{R}$. In general such functionals allow for a representation in terms of an integral $\Pi(\cdot)=\int \cdot \mathrm{d} \mu$. The main step is then to find the characterizing properties of those measures that define these integrals.

4.1. Standard Dual Space under Risk. We recall the well-known dual space representation of Lebesgue spaces under the uncertainty model described by a probability space $(\Omega, \mathcal{F}, P)$. In view of $(1)$, the classical Lebesgue space $L^{p}(P):=L^{p}(\Omega, \mathcal{F}, P)$ is then given by taking $\mathscr{P}=\{P\}$. The Riesz(-Frechet) representation theorem then establishes that the topological dual $L^{p}(P)^{*}$, where $p \in[1, \infty)$, can be identified by another Lebesgue space with conjugate order of integrability $\frac{1}{p}+\frac{1}{q}=1$ :

$$
L^{p}(P)^{*}=\left\{\Pi: L^{p}(P) \rightarrow \mathbb{R}: \Pi(\cdot)=E^{P}[\psi \cdot] \text { for a } \psi \in L^{q}(P)\right\} .
$$

Note that, for the space of progressively measurable $p$-integrable processes, dual elements of $L^{p}(\mathrm{~d} t \otimes P)$ can be represented by $\Pi(x)=E^{P}\left[\int_{0}^{T} \psi_{t} x_{t} \mathrm{~d} t\right], \psi \in L^{q}(\mathrm{~d} t \otimes P)$. 
4.2. Dual Space under Knightian Uncertainty. A meaningful modification of Lebesgue spaces is stated in Theorem 1 of Section 3 and denoted by $L^{p}(\mathscr{P})$, with $p \in[1, \infty)$. The Riesz-type representation for $\left(L^{p}(\mathscr{P}),\|\cdot\| \mathscr{P}, p\right)$ turns out to allow for a similar representation as in the case of classic Lebesgue spaces, as stated in (4).

Theorem 2. (Representation of the Dual Space) Let $\mathscr{P}$ be a weakly compact and convex set in $\Delta(\Omega)$. For any $p, q \in[1, \infty)$ with $\frac{1}{p}+\frac{1}{q}=1$, we have the following representation of the topological dual space

$$
L^{p}(\mathscr{P})^{*}=\left\{\Pi: L^{p}(\mathscr{P}) \rightarrow \mathbb{R}: \Pi(\cdot)=E^{P}\left[\psi_{P} \cdot\right] \text { for a } P \in \mathscr{P}, \psi_{P} \in L^{q}(P)\right\} .
$$

The proof is stated in Appendix B. [?] shows that any positive linear continuous functional on $L\left(c_{\mathbb{E}}^{p}\right)$ can be represented by a positive measure $\mu: \mathcal{F} \rightarrow \mathbb{R}_{+}$that does not charge polar sets, i.e. $\mu(A)=0$ for any $A \in \mathcal{N}$. Departing from this result, Theorem 2 shows that any representing $\mu$ allows for a decomposition of the form

$$
A \mapsto \mu(A)=\int_{A} \psi_{P}(\omega) \mathrm{d} P(\omega)
$$

The comparative statics work in the exact way as one may expect: Consider an increase in the degree of Knightian uncertainty from $\mathscr{P}$ to a larger set $\mathscr{P}^{\prime}$. Then, for any fixed $p \in[1, \infty)$, the norm $\|\cdot\|_{\mathscr{P}^{\prime}, p}$ is stronger, the dual norm becomes weaker and the dual space $L^{p}\left(\mathscr{P}^{\prime}\right)^{*}$ increases, given $L^{p}(\mathscr{P})^{*}$. Alternatively, this type of increase can be directly seen from the representation in (6), for some $P$ in $\mathscr{P}^{\prime}$.

Remark 2. In Proposition 2 of [?] it is only shown for the case $p=1$ that for every $P \in \mathscr{P}$ and $\psi \in L^{\infty}(P), \Pi(\cdot)=E^{P}[\psi \cdot]$ lies in $L^{1}(\mathscr{P})^{*}$.

4.3. Dual Space of Stochastic Processes. We now apply Theorem 2 to the space of progressively measurable stochastic processes under the filtration $\mathbb{F}=$ $\left(\mathcal{F}_{t}\right)_{t \in[0, T]}$, with $\mathcal{F}_{t}=\mathcal{B}\left(\Omega_{t}\right)$ and $\Omega_{t}=C_{0}[0, t]$. Let $\mathscr{P} \subset \Delta\left(\Omega_{T}\right)$ be weakly compact.

We work on the (polish) product space $\Omega=[0, T] \times \Omega_{T}$ and consider the set of product probability measures on $[0, T] \times \Omega_{T}$

$$
\mathscr{P}_{T}=\left\{\frac{1}{T} \mathrm{~d} t \otimes P \in \Delta(\Omega): P \in \mathscr{P}\right\},
$$

where $\mathrm{d} t$ is the Lebesgue measure on $[0, T]$. The set $\mathscr{P}_{T}$ is weakly compact if so $\mathscr{P}$, hence as a special case of Section 3 , we can construct $L^{p}\left(\mathscr{P}_{T}\right)$ for any $p \in[1,+\infty)$. Each $\eta \in L^{p}\left(\mathscr{P}_{T}\right)$ is a function of $(t, \omega) \rightarrow \eta(t, \omega)=\eta_{t}(\omega)$ with a norm is given by

$$
\|\eta\|_{L^{p}\left(\mathscr{P}_{T}\right)}=\frac{1}{T} \max _{P \in \mathscr{P}} E^{P}\left[\int_{0}^{T}\left|\eta_{s}\right|^{p} \mathrm{~d} s\right]^{1 / p} .
$$

An important class of processes (as a space of integrand for a stochastic analysis under volatility uncertainty) is given by

$$
M^{p}(\mathscr{P})=\left\{\eta \in L^{p}\left(\mathscr{P}_{T}\right) \mathbb{F} \text {-progressively measurable }\right\} .
$$

Clearly, $M^{p}(\mathscr{P})$ is a $\|\cdot\|_{L^{p}\left(\mathscr{P}_{T}\right)}$-closed subvector space of $L^{p}\left(\mathscr{P}_{T}\right)$.

Remark 3. If $\mathscr{P}$, as in Example 1.2, these Banach spaces of processes are wellknown in the $G$-expectation theory, see [?] for a result that constructs also $M^{p}(\mathscr{P})$ via a similar approach to method 2. 
As a corollary of Theorem 2 we can also represent the topological dual of the Banach space $M^{p}(\mathscr{P})$ :

Corollary 1. Let $p \in[1, \infty)$ and $q \in(1,+\infty]$ its conjugate then $\Pi: L^{p}\left(\mathscr{P}_{T}\right) \rightarrow$ $\mathbb{R}$ belongs to the topological dual space of $M^{p}(\mathscr{P})$ if and only if there exists $P \in \mathscr{P}$ and $\tilde{\psi^{P}}$, a progressively measurable process in $L^{q}([0, T] \times \Omega ; d t \otimes P)$, such that

$$
\forall \eta \in M^{p}(\mathscr{P}), \Pi(\eta)=E^{P}\left[\int_{0}^{T} \tilde{\psi}_{t}^{P} \eta_{s} \mathrm{~d} s\right] .
$$

Proof. Let $\Pi$ be in the topological dual space of $M^{p}(\mathscr{P})$. By the Hahn-Banach Theorem, there exists a $\hat{\Pi}$ in $L^{p}\left(\mathscr{P}_{T}\right)^{*}$ such that $\hat{\Pi}_{\mid M^{p}}=\Pi$. As a consequence of Theorem 2, there exist $P \in \mathscr{P}$ and $\psi^{P}$ in $L^{q}\left([0, T] \times \Omega_{T} ; \mathrm{d} t \otimes P\right)$ such that

$$
\forall \eta \in M^{p}(\mathscr{P}), \hat{\Pi}(\eta)=\Pi(\eta)=\frac{1}{T} E^{P}\left[\int_{0}^{T} \psi_{s}^{P} \eta_{s} \mathrm{~d} s\right] .
$$

Denoting by $\tilde{\psi}^{P}$ the progressively measurable projection of $\frac{1}{T} \psi^{P}$, the result is proved. $\square$

For the rest of the paper we exploit the impact of Theorem 2 and try to understand the implications for a general equilibrium theory under volatility uncertainty. In particular, we see in Section 5 that the representation result of Theorem 2 has far reaching consequences for a sound microeconomic foundation of finance under Knightian uncertainty.

5. Implications for General Equilibrium Theory. We recall the equilibrium notion under risk and fix $p=2$. The basic object of an economy (under uncertainty) refers to the commodity space of contingent claims, that is, the set of all payoffs $x: \Omega \rightarrow \mathbb{R}$ being available in the economy with $\mathbb{I}=\{1, \ldots, I\}$ agents.

5.1. The Case of Risk $\mathscr{P}=\{P\}$. Fix the commodity space of payoffs with finite variance $L^{2}(P)$ and the economy $\mathcal{E}^{P}=\left\{L^{2}(P)_{+}, U^{i}, e^{i}\right\}_{i \in \mathbb{I}}$, where the positive cone $L^{2}(P)_{+}$is the consumption set, $e^{i} \in L^{2}(P)_{+}$is the random endowment of agent $i$ and $U^{i}: L^{2}(P)_{+} \rightarrow \mathbb{R}$ denotes her utility functional, e.g. $U^{i}(\cdot)=E^{P}[\ln (\cdot)]$. We assume that at least one agent has strictly monotone preferences. ${ }^{4}$ The following notion of equilibrium is standard. ${ }^{5}$

Definition 1. $(\bar{c} ; \Pi) \in L^{2}(P)_{+}^{I} \times L^{2}(P)^{*}$ is an Arrow-Debreu equilibrium in $\mathcal{E}^{P}$ if

1. for all $i \in \mathbb{I}, \bar{c}^{i}$ maximizes $U^{i}$ on $\left\{x \in L^{2}(P)_{+}: \Pi\left(c-e^{i}\right) \leq 0\right\}$.

2. the allocation $\bar{c}$ is feasible: $\sum_{i \in \mathbb{I}} \bar{c}^{i}=\sum_{i \in \mathbb{I}} e^{i} P-$ a.s.

The equilibrium price $\Pi \in L^{2}(P)^{*}$ allows for an explicit description in terms of a state-price density $\psi \in L^{2}(P)$. As in Subsection 4.1 and (4), this yields the usual relationship through the dual pairing of the commodity and price space:

$$
\Pi(x)=\langle\psi, x\rangle=E^{P}[\psi x],
$$

for some $\psi \in L^{2}(P)$. Strictly monotone and concave utilities $U^{i}$ then guarantees $\psi>0$ $P$-a.s., by the first order condition of each optimization problem of Definition 1.1. We may normalize $\psi$, when considered as a Radon-Nikodym derivative and recover the classic equivalent martingale measure $(\mathrm{EMM}) \mathrm{d} \mathbb{Q}=\frac{\psi}{E^{P}[\psi]} \mathrm{d} P$. With zero interest rate, the equilibrium price $\Pi$ is then the expectation under the EMM: $\Pi(x)=E^{\mathbb{Q}}[x]$.

\footnotetext{
${ }^{4}$ I.e., there is some $k \in \mathbb{I}$, such that $U^{k}(c+d)>U^{k}(c)$, for any $C \in L^{2}(P)_{+}$and $d \in L^{2}(P)_{+} \backslash\{0\}$.

${ }^{5}$ For existence of equilibria, see the overview article [?].
} 
5.2. The Case of Knightian Uncertainty. To see the major change under volatility uncertainty, we fix a convex and weakly compact set $\mathscr{P}$ and set $L:=L^{2}(\mathscr{P})$, the space of random variables from Section 3. Note that since $L$ is a Banach lattice, linear and positive functionals on $L$ are automatically $\|\cdot\|_{\mathscr{P}, p}$-continuous.

We consider the following standard exchange economy under $\mathscr{P}$ :

$$
\mathcal{E}^{\mathscr{P}}=\left\{L_{+}, U^{i}, e^{i}\right\}_{i \in \mathbb{I}},
$$

where $e^{i} \in L_{+}:=\left\{x \in L: x \geq 0 \mathscr{P}_{\text {-q.s. }}\right\}$ is again the random endowment of the $i$-th agent and $U^{i}: L_{+} \rightarrow \mathbb{R}$ denotes the utility functional of agent $i$. The equilibrium concept under $\mathscr{P}$ is an adjusted version of Definition $1 .^{6}$

Definition 2. $(\bar{c} ; \Pi) \in L_{+}^{I} \times L^{*}$ is an Arrow-Debreu equilibrium in $\mathcal{E}^{\mathscr{P}}$, if

1. for all $i, \bar{c}^{i}$ maximizes $U^{i}$ on $L_{+}$under the constraint $\Pi\left(c-e^{i}\right) \leq 0$.

2. the allocation $\bar{c}$ is feasible: $\sum_{i \in \mathbb{I}} \bar{c}^{i}=\sum_{i \in \mathbb{I}} e^{i} \mathscr{P}-$ q.s.

Note that the feasibility condition in Definition 2 is now in the quasi sure sense.

With this slightly modified equilibrium notion, we may employ Theorem 2 and explore some properties of a linear equilibrium price system under volatility uncertainty.

COROLlary 2. The equilibrium determines a collection of relevant null events, induced by $P_{\Pi}$, i.e. any payoff $x \in L_{+}$outside the support of $P_{\Pi}$ is zero. More precisely, if $\{\omega \in \Omega: x(\omega)>0\} \cap \operatorname{supp}\left(P_{\Pi}\right)=\emptyset$ then $\Pi(x)=0$.

Proof. By (8), the equilibrium price system $\Pi$ is determined by the linear expectation under some $P_{\Pi} \in \mathscr{P}$. The possible mutual singularity to another $P^{\prime} \in \mathscr{P}$ gives an event $A \in \mathcal{F}$ that satisfies $P^{\prime}(A)>0$ and $P_{\Pi}(A)=0$. Consequently, $\Pi$ can only assign a strictly positive value to those payoffs that are not a $P_{\Pi \text {-null set. }}$

By Theorem 2, any equilibrium prices system is of the form

$$
\Pi(x)=E^{P_{\Pi}}\left[\psi_{P} x\right], \quad \text { for some } P_{\Pi} \in \mathscr{P} \text { and } \psi_{P} \in L^{2}\left(P_{\Pi}\right) .
$$

This representation has a similar form as the equilibrium price system for the economy $\mathcal{E}^{P}$ under sole risk in (7). However, in the present setting of possibly mutually singular probability measures, the functional form of $\Pi$ is a more delicate issue. For instance, the representation (8) creates an intrinsic weakness of linear equilibrium price systems. The price functional $\Pi: L \rightarrow \mathbb{R}$ can no longer be aware of any payoff outside the support of the representing probability measure $P_{\Pi}$.

Proposition 1 establishes existence of equilibrium for a class of standard utility functionals. Moreover, we observe a sharper description of the state-price density $\psi_{P}$.

Proposition 1. Let every agent $i \in \mathbb{I}$ in $\mathcal{E}^{\mathscr{P}}$ has a worst-case expected utility

$$
U^{i}(x)=\min _{P \in \mathscr{P}} E^{P}\left[u^{i}(x)\right]
$$

with strictly increasing, differentiable and strictly concave utility index such that $x \mapsto$ $x u^{i^{\prime}}(x)$ is increasing and $e:=\sum_{i} e^{i}$ is $\mathscr{P}_{-}$.s. is bounded away from zero. Then an equilibrium $\left(\bar{c}^{1}, \ldots, \bar{c}^{I}, \Pi\right)$ exists with $\Pi(\cdot)=E^{P_{\Pi}}\left[\psi_{P} \cdot\right]$ and the state-price density $\psi_{P}$ in (8) satisfies $\psi_{P} \in L_{+}$and $\psi_{P}>0$ q.s. For each $i, P_{\Pi}$ is a minimizer of $U^{i}$ at $\bar{c}^{i}$.

\footnotetext{
${ }^{6}$ This paper presents not another existence result of equilibrium. For such results see [?].
} 
Proof. Our conditions on the economy satisfy Assumption 1 in [?] and by Theorem 1 therein, we get existence of an equilibrium. Moreover, we get that $\psi_{P}=u_{\alpha}^{\prime}(e) P_{\Pi^{-}}$ a.s. Note that $\left(\alpha_{1}, \ldots, \alpha_{I}\right)$ is a Pareto weighting of the efficient equilibrium allocation and the aggregated utility index is defined by $u_{\alpha}(e)=\max _{\sum_{i} x^{i} \leq e, x^{i} \geq 0} \sum_{i} \alpha_{i} u^{i}\left(x^{i}\right)$ (for details we refer to Section 6.3.2 in [?]). Since $e$ is bounded away from zero and each $u^{i}$ is strictly increasing, concave and continuously differentiable, the same holds for $u_{\alpha}$, consequently $\left\|u_{\alpha}^{\prime}(e)\right\|_{\mathscr{P}, 2}<\infty$ and $u_{\alpha}^{\prime}(e)>0 \mathscr{P}_{\text {-q.s. }}$ Moreover, since $u_{\alpha}^{\prime}$ is continuous, which follows from the continuous differentiability of each $u^{i}$, it follows that $u_{\alpha}^{\prime}(e)$ is quasi-continuous and by Theorem 1 we have $\psi_{P} \in L^{2}(\mathscr{P})_{+} . \psi_{P}>0$ q.s. follows directly from the assumption on $e$ and since each $u^{i}$ is strictly increasing.

To see that $P_{\Pi}$ is for each agent a minimizer at her equilibrium consumption note that the first-order condition with respect to the first part of Definition 2 requires $\Pi \in \partial U^{i}\left(\bar{c}^{i}\right)$, the superdifferential of $U^{i}$ at $\bar{c}^{i}$ for any agent $i \in \mathbb{I}$. The result then follows from Proposition 4 in [?].

5.3. Examples. We illustrate some new aspects of Arrow-Debreu equilibra under volatility uncertainty. We begin with a case where disagreement in beliefs results in non existence of equilibria.

EXAMPLE 2. Let there be $I=2$ agents in the economy and let the uncertainty be that of Example 1 with constant volatility bounds $[\underline{\sigma}, \bar{\sigma}]$. Specifically, suppose each agent is overconfident with subjective expected utility, given by Gateaux differentiable and concave functionals $U^{1}(\cdot)=E^{P^{\sigma}}[\ln (\cdot)]$ and $U^{2}(\cdot)=E^{P^{\underline{\sigma}}}[\ln (\cdot)]$. Assume strictly

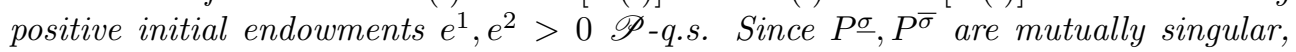
see Section 2, no equilibrium exists. To see this, we check the first-order conditions for optimality and get $\nabla U^{1}\left(\bar{c}^{1} ; \cdot\right)=\mu^{1} \Pi, \nabla U^{2}\left(\bar{c}^{2} ; \cdot\right)=\mu^{2} \Pi,{ }^{7}$ for some constants $\mu^{1}, \mu^{2} \geq 0$ and some equilibrium allocation $\left(\bar{c}^{1}, \bar{c}^{2}\right)$. But this can only be true if $\Pi=0 \in L^{*}$, since the utility gradients in $L^{*}$ satisfy $\nabla U^{1}\left(\bar{c}^{1} ; \cdot\right)=E^{P^{\bar{\sigma}}}\left[\frac{1}{\bar{c}^{1}} \cdot\right]$ and $\nabla U^{2}\left(\bar{c}^{2} ; \cdot\right)=E^{P^{\underline{a}}}\left[\frac{1}{\bar{c}^{2}} \cdot\right]$. On the other hand, we have by the shape of each $U^{i}, \bar{c}^{i}>0$ on the respective domain of the agent's belief, which implies $\nabla U^{i}\left(\bar{c}^{i} ; \cdot\right) \neq 0$, for $i=1,2$.

The example is a typical consequence of volatility uncertainty and the disagreement of null sets. In the case of drift uncertainty, priors are mutually equivalent and trade can be rather excessive, see [?] and the price for positive claim is not zero.

Agents in the economy of Example 3 share at least some priors in $\mathscr{P}$.

EXAMPLE 3. Let there be two pessimistic expected utility agents, with sets of beliefs $\mathscr{P}_{1}, \mathscr{P}_{2} \subset \mathscr{P}$. We can write this in terms of variational preferences, see [?], and get

$$
U^{i}(c)=\min _{P \in \mathscr{P}} E^{P}[\ln (c)]+c^{i}(P)
$$

where $\mathrm{c}^{i}: \mathscr{P} \rightarrow[0, \infty]$ is zero if $P \in \mathscr{P}^{i}$ and otherwise $+\infty$.

Let us assume that $e^{i}>0$ q.s. and $\mathscr{P}^{1} \cap \mathscr{P}^{2} \neq \emptyset$ then we get from Theorem 1 of [?] existence of an Arrow-Debreu equilibrium in $\mathcal{E}^{\mathscr{P}}=\left\{L^{2}(\mathscr{P})_{+}, U^{i}, e^{i}\right\}_{i=1,2}$. As long as agents share a prior, this result holds for more than two agents.

As a preparation of Section 6, we now consider a single-agent economy. As trade will not occur, the insights from a no-trade equilibrium will only be revealed by the properties of the equilibrium price system $\Pi$. This enables us to investigate the principle pricing mechanisms, by recasting the notion of viability from [?].

\footnotetext{
${ }^{7}$ By the assumptions on $e^{i}$, the Slater conditions (with respect to the problems in Definition 2.1 ) holds for any agent and any positive and nonzero $\Pi \in L^{*}$.
} 
ExAMPLE 4. Again, we depart from the uncertainty model of Example 1. Let there be a single agent in a no-trade equilibrium with some initial endowment. Moreover, suppose that this agent has maxmin expected utility in the sense of [?]:

$$
U(c)=\min _{P \in \mathscr{P}} E^{P}[u(c)], \quad \text { for any } c \in L_{+},
$$

where $u: \mathbb{R} \rightarrow \mathbb{R}$ is assumed to be increasing, continuously differentiable and concave. Let $e=\left|B_{T}\right|$. Since $U(c)=-\mathbb{E}[-u(c)]$, we have $U(e)=E^{P^{\bar{\sigma}}}[u(e)]$, since $-u \circ|\cdot|$ is convex. Pessimism lets the agent consider the maximal volatility regime, since endowment is a convex function of the $G$-Brownian motion. ${ }^{8}$

$A$ viable price system $\Pi$, making the representative agent economy into a notrade equilibrium, is then given by $x \mapsto \Pi(x)=E^{P^{\bar{\sigma}}}\left[u^{\prime}(e) x\right]$. Since $u^{\prime}$ is continuous it follows by Theorem 1 that $u^{\prime}(e) \in L^{2}(\mathscr{P}) \subset L^{2}\left(P^{\bar{\sigma}}\right)$. But by Theorem 2, we then have $\Pi \in L^{2}(\mathscr{P})^{*}$. The form of $\Pi$ follows from the necessary and sufficient first-order condition of the constrained optimization problem in Definition 2.1 for the case $I=1$.

Consequently, $\Pi$ is only capable to detect positive payoffs within the support of $P^{\bar{\sigma}}$, the prior under which the volatility is maximal.

In Section 6, we focus on the standard approach to asset pricing via equivalent martingale measures and the relation to a no-arbitrage pricing principle.

6. Implications for the Foundations of Finance. The discussion of the last section has direct and far-reaching consequences for an asset pricing theory that builds on principles of Arrow-Debreu equilbria, introduced in Definition 2. Especially Example 4 makes this point clear.

6.1. Static Asset Pricing. The next definition is a robust counterpart to no arbitrage under risk.

Definition 3. (no-arbitrage) A linear and positive price system $\Pi: L \rightarrow \mathbb{R}$ is $\mathscr{P}$-arbitrage free if there is no costless and nontrivial positive payoff $X: \Omega \rightarrow \mathbb{R}$, that is, $X \in L_{+} \backslash\{0\}$, such that $\Pi(X)=0$.

From a geometric perspective the arbitrage cone coincides with the positive cone with deleted zero. With Definition 3, an arbitrage for some nonnegative payoff is already present, when there is at least one volatility model $P^{\sigma}$ under which an arbitrage in the classical sense occurs. This follows from the fact that $X \in L_{+} \backslash\{0\}$ implies $P(X>0)>0$ for some $P \in \mathscr{P}$ and $X \geq 0 \mathscr{P}$-quasi surely.

For the rest of this section, let the uncertainty be given by Peng's G-expectation and the related interval $[\underline{\sigma}, \bar{\sigma}]$, see Example 1.2 for details.

Corollary 3. For any nonzero positive price system $\Pi: L \rightarrow \mathbb{R}$, we can find an arbitrage opportunity.

Proof. By Theorem 2, we have $\Pi(X)=E^{P}[\psi X]$ for some nonzero $\psi \in L^{2}(P)_{+}$ and $P \in \mathscr{P}$. As discussed in Example 5, we can find some $Y_{P} \in L_{+} \backslash\{0\}$ which is only non-zero outside the support of the a priori given $P \in \mathscr{P}$. It then directly follows that $Y_{P}$ is an arbitrage with respect to the price system $\Pi$ and the result follows.

This simple result tells us that the existence of an equivalent martingale measure $Q \in \Delta(\Omega)$, considered as a normalized version of $\Pi$, see Theorem 1 of [?], is no longer sufficient for excluding arbitrage in a financial market.

\footnotetext{
${ }^{8}$ See [?] and [?] for an exposition.
} 
REMARK 4. Corollary 3 is at first glance a bit surprising. However, as shown in [?] or [?] in the case of the fundamental theorem of asset pricing (FTAP) under volatility uncertainty, to exclude $\mathscr{P}$-arbitrage there has to exist a set of mutually singular pricing measure. In contrast to pricing under single prior uncertainty, a linear pricing measure can no longer exclude the present type of strong arbitrage. In Subsection 6.2, we derive such a set.

The following example presents a specific case in which certain positive volatility derivatives are costless when priced under some equilibrium price system $\Pi \in L_{+}^{*}$. In the proof of Corollary 3 the existence of such a claim is needed.

EXAmPle 5. Let $P_{\Pi}=P^{\bar{\sigma}}$ be the "equilibrium" prior of the equilibrium price $\Pi \in L^{*}$. In the case of an economy with ambiguity averse agents, such an equilibrium outcome is likely to happen, see Example 4. Assume that the financial market follows a modified Bachelier model under ambiguous volatility, that is the risky (and ambiguous) asset follows a $G$-Brownian motion $\left(B_{t}^{G}\right) .{ }^{9}$

Consider a claim of the form $X=1_{A}$ on the event $A=\left\{\omega:\left\langle B^{G}\right\rangle_{T}(\omega) \in\right.$ $\left.\left[\underline{\sigma}, \underline{\sigma}+\frac{\bar{\sigma}-\underline{\sigma}}{2}\right]\right\}$. The claim $X$ is then a volatility derivative of digital type.

In general, $X \notin L^{2}(\mathscr{P})$ since it is not quasi-continuous. Nevertheless, we can approximate $X$ in the following way: consider $\left(\varphi_{n}\right)_{n \geq 1}$ a non increasing sequence of nonnegative continuous function, converging everywhere to $1_{\left[\underline{\sigma}, \frac{\sigma+\sigma}{2}\right]}$ such that $\varphi_{n}(x)=1$ if $x \in\left[\underline{\sigma}, \frac{\bar{\sigma}+\underline{\sigma}}{2}\right], \varphi_{n}(x)=0$ if $x \leq \underline{\sigma}-\frac{1}{n}$ or $x \geq \frac{\bar{\sigma}+\underline{\sigma}}{2}+\frac{1}{n}$. We set $X_{n}=\varphi_{n}\left(\left\langle B^{G}\right\rangle_{T}\right) \in$ $L^{2}(\mathscr{P})$, then it is clear that $X_{n} \geq X$ q.s. Moreover, for $n$ large enough $E^{P^{\bar{\sigma}}}\left[X_{n}\right]=0$, hence it turns out that in equilibrium such contingent claims are for free, since it implies $\Pi\left(X_{n}\right)=E^{P_{\Pi}}\left[\psi_{P_{\Pi}} X_{n}\right]=0$. This implies that the price of $X$ has to be zero.

This example illustrates a critical aspect for a foundational role of Arrow-Debreu equilibria under volatility uncertainty. When seeking a theory of value for security prices under volatility uncertainty, Example 5 captures the shortcomings of linear equilibrium-price systems in a particular, but not pathological, case. This aspect plays a pivotal role, as the whole neoclassic finance theory ultimately relies on the Arrow-Debreu equilibrium concept. In particular, nearly every dynamic asset pricing theory relies on a marginal utility description of the universal system of equilibrium conditions (feasibility, budget constraints and first-order conditions).

Within the no-arbitrage paradigm, there is a risk-neutral pricing measure. The mutual equivalence with respect to the usually single physical probability, i.e. $\mathscr{P}=$ $\{\mathbb{P}\}$, yields the existence of a stochastic-discount factor that relates any payoff to asset prices in the economy.

6.2. Continuous Time Asset Pricing. By virtue of Subsection 4.3, we extend the asset pricing application to the continuous time setup. In view of Remark 4, consider the continuous time $G$-expectation setting of Example 1.2.

Let there be one agent with positive endowment process $\frac{\mathrm{d} e_{t}}{e_{t}}=\mu_{t}^{e} \mathrm{~d} t+V_{t}^{e} \mathrm{~d} B_{t}^{G}$, with $e_{0}=1$, where $\left(\mu_{t}^{e}\right),\left(V_{t}^{e}\right) \in M^{2}(\mathscr{P})_{+}=\left\{x \in M^{2}(\mathscr{P}): x_{t} \geq 0 \forall t\right.$ q.s. $\}$ are assumed to be bounded, with $V_{t}^{e}>0$ q.s. The utility functional $U: M^{2}(\mathscr{P})_{+} \rightarrow \mathbb{R}$ is as in [?]:

$$
U(c)=\min _{P \in \mathscr{P}} E^{P}\left[\int_{0}^{T} e^{-\beta t} u\left(c_{t}\right) \mathrm{d} t\right]
$$

where $\beta>0$ is a discount factor and $u: \mathbb{R} \rightarrow \mathbb{R}$ is a strictly increasing, three

\footnotetext{
${ }^{9}$ Note the result remains valid when the asset price follows a positive G-Ito process.
} 
times continuously differentiable and concave utility index. Usual arguments allows to consider a no-trade equilibrium of a financial market $\left(S^{0}, S^{1}\right)$ with locally riskless asset $S^{0}$ and uncertain asset $S^{1}$ satisfying

$$
\mathrm{d} S_{t}^{0}=S_{t}^{0} r_{t} \mathrm{~d} t, \quad \mathrm{~d} S_{t}^{1}=S_{t}^{1}\left(\mu_{t} \mathrm{~d} t+\Sigma_{t} \mathrm{~d} B_{t}^{G}\right)
$$

where the interest rate $r_{t}$, and the return $\mu_{t}$ are determined from the equilibrium. For simplicity set $\Sigma_{t}=1$, so that all the volatility uncertainty is contained in $B^{G}$.

By the concavity of $U$, the compactness of $\mathscr{P}$ and Corollary 2, there is a $P^{e} \in \mathscr{P}$ such that, $\nabla U(c ; \cdot) \in M^{2}(\mathscr{P})^{*}$ is a super gradient of $U$ at $e$ in direction $h \in M^{2}(\mathscr{P})$, given by

$$
\nabla U(c ; h)=E^{P^{e}}\left[\int_{0}^{T} e^{-\beta t} u^{\prime}\left(e_{t}\right) h_{t} \mathrm{~d} t\right]
$$

with the refinement $\left\{u^{\prime}\left(e_{t}\right)\right\} \in M^{2}(\mathscr{P}) \subset L^{2}\left(\mathrm{~d} t \otimes P^{e}\right)$. In particular, we may apply the Ito formula for $G$-Ito process to $\psi_{t}=e^{-\beta t} u^{\prime}\left(e_{t}\right)$ and get

$$
\frac{\mathrm{d} \psi_{t}}{\psi_{t}}=-\underbrace{\left(\beta+\frac{u^{\prime \prime}\left(e_{t}\right)}{u^{\prime}\left(e_{t}\right)} \mu_{t}^{e}-\frac{1}{2} u^{\prime \prime \prime}\left(e_{t}\right)\left(V_{t}^{e}\right)^{2}\right)}_{=r_{t}} \mathrm{~d} t-\underbrace{e_{t} \frac{u^{\prime \prime}\left(e_{t}\right)}{u^{\prime}\left(e_{t}\right)} V_{t}^{e}}_{=\theta_{t}} \mathrm{~d} B_{t}^{G}
$$

where $\theta_{t}$ denotes the market price of risk and ambiguity, see Section 3.1 in [?]. Consequently we have $\psi_{t}=\mathcal{E}_{t}^{\theta} \cdot e^{-\int_{0}^{t} r_{s} d s}$, where $\mathcal{E}_{t}^{\theta}$ is an exponential martingale under any $P \in \mathscr{P}$, solving the $G$-SDE $\mathrm{d} \mathcal{E}_{t}^{\theta}=-\mathcal{E}_{t}^{\theta} \theta_{t} \mathrm{~d} B_{t}^{G}, \mathcal{E}_{0}^{\theta}=1$ with explicit solution:

$$
\mathcal{E}_{t}^{\theta}=\exp \left(-\int_{0}^{t} \theta_{s} \mathrm{~d} B_{s}^{G}-\frac{1}{2} \int_{0}^{t} \theta_{s}^{2} \mathrm{~d}\left\langle B^{G}\right\rangle_{s}\right)
$$

By a classic change of measure and Fubini's theorem under $P^{e}$, we get the equilibrium price for any payoff stream $x=\left(x_{t}\right) \in M^{2}(\mathscr{P})$ :

$$
\Pi(x)=E^{P^{e}}\left[\int_{0}^{T} \mathcal{E}_{t}^{\theta} e^{-\int_{0}^{t} r_{s} d s} x_{t} \mathrm{~d} t\right]=E^{Q^{e}}\left[\int_{0}^{T} e^{-\int_{0}^{t} r_{s} d s} x_{t} \mathrm{~d} t\right] .
$$

Similarly to Corollary 3 , we may find an arbitrage if the pricing only relies on $\Pi$. In view of Remark 4, we can define a robust set of equilibrium pricing measures, that is based on $\mathcal{E}^{\theta}$ and $\mathscr{P}$. Define the following set of (mutually singular) pricing measures

$$
\mathscr{Q}=\left\{Q \in \Delta(\Omega): \mathrm{d} Q=\mathcal{E}_{T}^{\theta} \mathrm{d} P \text { for some } P \in \mathscr{P}\right\} .
$$

Under the "uncertainty neutral" expectation, see [?],

$$
\mathbb{E}^{\theta}\left[e^{-\int_{0}^{T} r_{s} d s} X\right]=\mathbb{E}\left[e^{-\int_{0}^{T} r_{s} d s} \mathcal{E}_{T}^{\theta} X\right]
$$

on $L^{2}(\mathscr{P})$, the asset prices $S^{0}$ and $S^{1}$ are then $Q$-martingales under every $Q \in$ $\mathscr{Q}$. From this, we see that the connection between equilibria and the FTAP under volatility uncertainty are less tight as in the case of sole risk.

REMARK 5. The novel effect under uncertain volatility relies on the equilibrium prior that is the endogenous outcome of the equilibrium. In models under sole risk, as in Subsection 5.1, all relevant data for pricing can be summarized by the state-price density or the stochastic-discount factor. Under uncertain volatility this is no longer possible. One immediate consequence is that, the tight connection between single agent 
no-trade equilibria with a utility gradient approach and a consumption-based asset pricing model, such as in [?], appear in a different light. ${ }^{10}$

7. Conclusion. This paper establishes an infinite-dimensional commodity space in the presence of a fairly general form of Knightian uncertainty. In a second step we fully describe the topological dual space. Our application to general equilibrium theory relies on the explicit representation of any linear and continuous pricing functional on the new commodity space. We show that linear price systems can only detect a subclass of favorable payoffs. In a further discussion we move then to some direct implications for standard asset pricing. For instance, it turns out that linear price systems allow for arbitrage opportunities under volatility uncertainty.

A further line of application points to convex risk measures and monetary utility functions under volatility uncertainty. Theorem 2 allows then to represent concave/convex functionals by means of the standard Legendre-Fenchel Duality.

Appendix A. Capacity related to a sublinear expectation. We collect results to connect Methods 1-3 from Section 3. As the proof of Theorem 2 in Appendix B relies on Method 3, we provide some preparation. Let $\Omega$ be a polish space.

Definition 4. A functional $\hat{\mathbb{E}}: \mathcal{H} \mapsto \mathbb{R}$ is called a sublinear expectation on a vector lattice $\mathcal{H}$ of functions, with $1 \in \mathcal{H}$ and $C_{b}(\Omega) \subset \mathcal{H}$ if we have for all $X, Y \in \mathcal{H}$ :

1. Monotonicity: $X \geq Y$ implies $\hat{\mathbb{E}}[X] \geq \hat{\mathbb{E}}[Y]$.

2. Sub-additivity: $\quad \hat{\mathbb{E}}[X+Y] \leq \hat{\mathbb{E}}[X]+\hat{\mathbb{E}}[Y]$.

3. Positive homogeneity: for all $\lambda \geq 0, \quad \hat{\mathbb{E}}[\lambda X]=\lambda \hat{\mathbb{E}}[X]$.

4. Constant translatability: for all $c \in \mathbb{R}, \quad \hat{\mathbb{E}}[X+c]=\hat{\mathbb{E}}[X]+c$.

This section consider a sublinear expectation $\hat{\mathbb{E}}: \mathcal{H} \rightarrow \mathbb{R}$. We assume that $\mathcal{H}$ generates the topology of $\Omega$ and also that $\hat{\mathbb{E}}$ is regular, i.e., for any decreasing sequence $\left(X_{n}\right)_{n \in \mathbb{N}}$ in $\mathcal{H}$ tending to 0 everywhere on $\Omega$, we have $\lim _{n} \hat{\mathbb{E}}\left[X_{n}\right]=0$. We prove that $\hat{\mathbb{E}}$ is the upper expectation associated to a weakly compact family $\mathscr{P}$.

For all $X \in \mathcal{H}$, we set $c_{\hat{\mathbb{E}}}^{p}(X):=\hat{\mathbb{E}}\left[|X|^{p}\right]^{\frac{1}{p}}$. Clearly, $c_{\hat{\mathbb{E}}}^{p}$ is a semi-norm on $\mathcal{H}$ such that $c_{\widehat{\mathbb{E}}}^{p}(1)=1$ and $c_{\widehat{\mathbb{E}}}^{p}(X)=c_{\widehat{\mathbb{E}}}^{p}(|X|)$, hence, $c_{\widehat{\mathbb{E}}}^{p}(X) \leq 1$ if $|X| \leq 1$. The Lebesgue extension of $c_{\hat{\mathbb{E}}}^{p}$ is introduced in Method 3 in Subsection 3.1. For $A \subset \Omega$ we put

$$
\mathbb{C}(A):=c_{\hat{\mathbb{E}}}^{1}\left(\mathbf{1}_{A}\right) .
$$

Regularity of $\hat{\mathbb{E}}$ implies that $\mathbb{C}$ is a Choquet capacity, see [?], Proposition 11.

TheOREM 3. The set function $A \mapsto \mathbb{C}(A)$ is a Choquet capacity, i.e.,

1. $0 \leq \mathbb{C}(A) \leq 1$, for each $A \in \mathcal{B}(\Omega)$.

2. If $A \subset B$, then $\mathbb{C}(A) \leq \mathbb{C}(B)$.

3. If $\left(A_{n}\right)$ is a sequence of sets in $\mathcal{B}(\Omega)$, then $\mathbb{C}\left(\cup_{n} A_{n}\right) \leq \sum_{n} \mathbb{C}\left(A_{n}\right)$.

4. For any $\varepsilon>0$, there exists a compact set $K \subset \Omega$ such that $\mathbb{C}(\Omega \backslash K) \leq \varepsilon$.

We still use the standard capacity-related vocabulary: a set $A$ is $\mathbb{C}$-polar if $\mathbb{C}(A)=0$ and a property holds "CC-quasi-surely" (q.s.) if it holds outside a polar set. In view of (9), we may also define $\mathbb{C}^{p}$ via $c_{\widehat{\mathbb{E}}}^{p}$ for any $p \in[1, \infty)$. However, polor sets are independent of $p \in[1, \infty)$, since $\mathbb{C}^{1}(A)=0$ if and only if $\mathbb{C}^{p}(A)=0$.

\footnotetext{
${ }^{10}$ In their Subsection 3.3, a discussion on the difference between minimizing and dynamically minimizing measures for maxmin expected utility can be found. As discussed in Example 6, minimizing measure have a direct relation to the representation price systems of Arrow-Debreu equilibria.
} 
A.1. Extension of $\hat{\mathbb{E}}$. Thus far, $\hat{\mathbb{E}}$ is only defined on $\mathcal{H}$. Following [?], we first extend $c_{\widehat{\mathbb{E}}}^{p}$. For this, we denote by $\mathcal{L}\left(c_{\hat{\mathbb{E}}}^{p}\right)$ the closure of $\mathcal{H}$ in $\left\{g \in L(\Omega): c_{\hat{\mathbb{E}}}^{p}(g)<\infty\right\}$, via the Lebesgue prolongation from Method 3.

TheOREM 4. For any $p \in[1, \infty)$, each $X \in \mathcal{L}\left(c_{\hat{\mathbb{E}}}^{p}\right)$ can be identified with a function on $\Omega$ defined q.s. Denote by $L\left(c_{\hat{\mathbb{E}}}^{p}\right)$ the quotient of $\mathcal{L}\left(c_{\widehat{\mathbb{E}}}^{p}\right)$ with respect to the quasi-sure equivalence relation, then $\left(L\left(c_{\hat{\mathbb{E}}}^{p}\right), c_{\hat{\mathbb{E}}}^{p}\right)$ is a Banach space. Moreover, $C_{b}(\Omega) \subset \mathcal{L}\left(c_{\hat{\mathbb{E}}}^{p}\right)$.

Proof. For details see [?] p. 880.

As in the case for polar sets, quasi-continuity is independent of $p \in[1, \infty)$.

Definition 5. A mapping $X: \Omega \rightarrow \mathbb{R}$ is quasi-continuous (q.c.) if $\forall \varepsilon>0$, there exists an open set $O$ with $\mathbb{C}(O)<\varepsilon$ such that $\left.X\right|_{O^{c}}$ is continuous. We say that $X: \Omega \rightarrow \mathbb{R}$ has a q.c. version if there exists a q.c. $Y: \Omega \rightarrow \mathbb{R}$ with $X=Y \mathbb{C}$-q.s.

Proposition 2. Let $\left(X_{n}\right)$ be a $c_{\hat{\mathbb{E}}}^{p}$-Cauchy sequence in $\mathcal{H}$. Then there exists a subsequence $\left(X_{n_{k}}\right)$ with: for each $\varepsilon>0$, there exists an open set $O$ with $c_{\hat{\mathbb{E}}}^{p}(O)<\varepsilon$ and $\left(X_{n_{k}}\right)$ converges uniformly on $O^{c}$. Each $X \in \mathcal{L}\left(c_{\widehat{\mathbb{E}}}^{p}\right)$ admits a q.c. version $\tilde{X}$.

Proof. We can copy the proof of Theorem 25 in [?] or Theorem 2.1 in [?].

We now extend $\hat{\mathbb{E}}$ to $\mathbb{E}$ in an easy way.

Proposition 3. For any $p \in[1, \infty), \hat{\mathbb{E}}$ can be extended uniquely to a continuous mapping from $\mathcal{L}\left(c_{\widehat{\mathbb{E}}}^{p}\right)$ to $\mathbb{R}$, denoted by $\mathbb{E}$ and we have $c_{\mathbb{E}}^{1}(X)=\mathbb{E}[|X|]$ for all $X \in \mathcal{L}\left(c_{\widehat{\mathbb{E}}}^{p}\right)$.

Moreover, $\mathbb{E}$ is a sublinear expectation on $\mathcal{L}\left(c_{\hat{\mathbb{E}}}^{p}\right)$, in the sense that it satisfies all the four properties of Definition 4 on $\mathcal{L}\left(c_{\mathbb{E}}^{p}\right)$.

Proof. All is clear and based on the fact that $X \rightarrow X^{+}$and $X \rightarrow|X|$ are contraction on $\mathcal{L}\left(c_{\mathbb{E}}^{p}\right)$. For details, we refer to [?].

A.2. The abstract dual space of $L\left(c_{\mathbb{E}}^{p}\right)$. An important analogy with classical measure theory is the representation property of the dual space of $L\left(c_{\mathbb{E}}^{p}\right)$, i.e., the quotient space of $\mathcal{L}\left(c_{\mathbb{E}}^{p}\right)$ with respect to the $\mathbb{C}$-quasi sure equivalence relation.

Proposition 4. If $l: L\left(c_{\mathbb{E}}^{p}\right) \rightarrow \mathbb{R}$ belongs to the topological dual space of $L\left(c_{\mathbb{E}}^{p}\right)$, then there exists a Radon measure $\mu$ on $\Omega$ which does not charge $\mathbb{C}$-polar sets with

$$
l(X)=\int_{\Omega} \tilde{X}(\omega) \mathrm{d} \mu(\omega), \forall X \in L\left(c_{\mathbb{E}}^{p}\right) .
$$

Proof. This is Proposition 11 in [?].

This Radon-property for elements in $L\left(c_{\mathbb{E}}^{p}\right)$ in Proposition 4 leads to the following representation of $\mathbb{E}$, which is a first step to prove Theorem 2 .

Theorem 5. Let $\mathcal{M}^{1}(\mathbb{E}) \subset \Delta(\Omega)$ do not charge $\mathbb{C}$-polar sets and be dominated by $\mathbb{E}$, i.e., $\int_{\Omega} X(\omega) \mathrm{d} P(\omega) \leq \mathbb{E}[X]$, for all $X \in C_{b}(\Omega), P \in \mathcal{M}^{1}(\mathbb{E})$. Then

$$
\mathbb{E}[X]=\sup _{P \in \mathcal{M}^{1}(\mathbb{E})} E^{P}[X], \quad \forall X \in L\left(c_{\mathbb{E}}^{1}\right) .
$$

Moreover, the map $\mathbb{E} \mapsto \mathcal{M}^{1}(\mathbb{E})$ is a bijection between the set of regular sublinear expectations and the set of convex compact sets of probabilities.

Proof. As a consequence of the Hahn-Banach Theorem, we have for all $X \in L\left(c_{\mathbb{E}}^{1}\right)$ :

$$
\mathbb{E}[X]=\sup _{l \in \mathscr{Q}} l(X), \quad \mathscr{Q}=\left\{l: L\left(c_{\mathbb{E}}^{1}\right) \rightarrow \mathbb{R}: \text { linear, } l(X) \leq \mathbb{E}[X] \forall X \in L\left(c_{\mathbb{E}}^{1}\right)\right\} .
$$


Let $l \in \mathscr{Q}$, then for all non-negative $X$, we get $-l(X)=l(-X) \leq \mathbb{E}[-X] \leq \mathbb{E}[0]=0$ so that $l$ is positive. Since any $l \in \mathscr{Q}$ is dominated by $\mathbb{E}$, we deduce the $c_{\mathbb{E}}^{1}$-continuity of $l$. Moreover: $l(1) \leq \mathbb{E}[1]=1$ and $-l(1)=l(-1) \leq \mathbb{E}[-1]=-1$, so that $l(1)=1$. As a consequence of Proposition 4, there exists a probability $P$, not charging polar sets, such that for all $X \in L\left(c_{\mathbb{E}}^{1}\right), l(X)=E^{P}[X]$. This yields

$$
\mathbb{E}[X] \leq \sup _{P \in \mathcal{M}^{1}(\mathbb{E})} E^{P}[X], \quad \forall X \in L\left(c_{\mathbb{E}}^{1}\right) .
$$

The converse inequality is obvious and we have the first part of the theorem.

The second assertion is a consequence of Theorem 12 in [?]. In particular,

$$
\mathbb{E}[X]=\sup _{P \in \mathcal{M}(\mathbb{E})} E^{P}[X], \quad \forall X \in C_{b}(\Omega),
$$

where $\mathcal{M}(\mathbb{E})$ denotes the sets of measure om $(\Omega, \mathcal{B}(\Omega))$ dominated by $\mathbb{E}$. Moreover they established that the map $\mathbb{E} \mapsto \mathcal{M}(\mathbb{E})$ is a bijection between the set of regular functional capacities and the set of weakly compact convex and hereditary (in the sense $P \in \mathcal{M}(\mathbb{E}) \Rightarrow \alpha P \in \mathcal{M}(\mathbb{E}) \forall \alpha \in[0,1])$ sets of measures. As a consequence of the proof of the first assertion, we know that in the case of a regular non-linear expectation the supremum in $(11)$ is attained on the subset $\mathcal{M}^{1}(\mathbb{E}) \subset \mathcal{M}(\mathbb{E})$. Then it is easy to conclude since the map $\mathcal{M}(\mathbb{E}) \mapsto \mathcal{M}^{1}(\mathbb{E})$ is clearly a bijection.

A.3. Links between $c_{\mathbb{E}}^{1}$ and the upper-capacity of $\mathscr{P}$. Appendix A.1 departs from a regular sub-linear expectation $\mathbb{E}$ and relates a weakly compact set $\mathcal{M}^{1}(\mathbb{E}) \subset \Delta(\Omega)$ via $(10)$ and associates the capacity norm $c_{\mathbb{E}}^{1}$ from (9). However, [?] take a converse perspective: starting from a weakly compact $\mathscr{P}$, inducing a regular sublinear expectation $\mathbb{E}$ given by

$$
\mathbb{E}[X]=\sup _{P \in \mathscr{P}} E^{P}[X], \quad \forall X \in C_{b}(\Omega)
$$

and extended by completion. To this end, we have introduced another capacity which is the upper probability $\mathbb{C}_{\mathscr{P}}$ given by

$$
\mathbb{C}_{\mathscr{P}}(A)=\sup _{P \in \mathscr{P}} P(A), \forall A \in \mathcal{B}(\Omega) .
$$

From now on, we consider a set $\mathscr{P} \subset \Delta(\Omega)$ that is convex and weakly compact. We denote by $\mathbb{E}$ the regular sublinear expectation defined by (12) and $\mathbb{C}^{1}=\mathbb{C}$ the capacity associated with $\mathbb{E}$ via $c_{\mathbb{E}}^{1}$ from $(9)$ and $\mathbb{C}_{\mathscr{P}}$ as above. As in Section $\mathrm{A}, \mathcal{L}\left(c_{\mathbb{E}}^{p}\right)$ still denotes the closure of $\mathcal{H}$ with respect to $c_{\mathbb{E}}^{p}$. We discuss the relations between the capacities $\mathbb{C}$ and $\mathbb{C}_{\mathscr{P}}$ and the different notions of quasi-continuity.

Proposition 5. 1. If $O$ is open, $\mathbb{C}(O)=\mathbb{C}_{\mathscr{P}}(O)$.

In particular, $\mathbb{C}$-quasi-continuity and $\mathbb{C}_{\mathscr{P}}$-quasi-continuity coincide.

2. We have $\mathbb{C}_{\mathscr{P}}(A) \leq \mathbb{C}(A)$, for all $A \in \mathcal{B}(\Omega)$.

3. If $K$ is compact then: $\mathbb{C}(K)=\mathbb{C}_{\mathscr{P}}(K)$.

4. Let $X: \Omega \rightarrow \mathbb{R}$ be q.c., then for all $\alpha \in \mathbb{R} \mathbb{C}(\{X>\alpha\})=\mathbb{C}_{\mathscr{P}}(\{X>\alpha\})$. In particular, let $X, Y$ be $\mathbb{C}$-q.c., then $X=Y \mathbb{C}$-q.s. $\Leftrightarrow X=Y \mathbb{C}$-q.s.

Proof. 1. Since $O$ is open, we can construct an increasing sequence $\left(\psi_{n}\right)_{n}$ of nonnegative functions in $C_{b}(\Omega)$ converging everywhere to $1_{O}$. We can also construct an increasing sequence $\left(\zeta_{n}\right)_{n}$ of nonnegative functions in $C_{b}(\Omega)$ such that $\mathbb{C}(O)=$ 
$\lim _{n \rightarrow+\infty} \mathbb{E}\left[\zeta_{n}\right]$. Set $\varphi_{n}=\psi_{n} \vee \zeta_{n} \geq 0$, so that $\left(\varphi_{n}\right)$ is an increasing sequence in $C_{b}(\Omega)$ converging everywhere to $1_{O}$ and such that $\mathbb{C}(O)=\lim _{n \rightarrow \infty} \mathbb{E}\left[\varphi_{n}\right]$. This yields:

$$
\mathbb{C}(O)=\sup _{n} \mathbb{E}\left(\varphi_{n}\right)=\sup _{n} \sup _{P \in \mathscr{P}} E^{P}\left[\varphi_{n}\right]=\sup _{P \in \mathscr{P}} \sup _{n} E^{P}\left[\varphi_{n}\right]=\sup _{P \in \mathscr{P}} P(O) .
$$

2. Let $O \supset A$ be open. By 1., we have $\mathbb{C}_{\mathscr{P}}(A) \leq \mathbb{C}_{\mathscr{P}}(O)=\mathbb{C}(O)$. This gives the result.

3. By part 2 , we know that $\mathbb{C}(K) \geq \mathbb{C}_{\mathscr{P}}(K)$. By standard arguments, we can construct a decreasing sequence of open sets, $\left(O_{n}\right)$, such that

$$
\bigcap_{n} O_{n}=K, \mathbb{C}(K)=\lim _{n} \mathbb{C}\left(O_{n}\right) \text {. }
$$

As $\mathscr{P}$ is compact and $\left(\overline{O_{n}}\right)$ is a decreasing sequence of closed sets with $\bigcap_{n} \overline{O_{n}}=K$ :

$$
\mathbb{C}_{\mathscr{P}}(K)=\lim _{n} \mathbb{C}_{\mathscr{P}}\left(\bar{O}_{n}\right) \geq \lim _{n} \mathbb{C}_{\mathscr{P}}\left(O_{n}\right)=\lim _{n} \mathbb{C}\left(O_{n}\right)=\mathbb{C}(K) .
$$

4. Let $\varepsilon>0$. By definition, there exists an open set $O \subset \Omega$ with $\mathbb{C}(O)=\mathbb{C}_{\mathscr{P}}(O)<\varepsilon$ such that $X$ is continuous on $O^{c}$. As a consequence, $\{X>\alpha\} \cap O^{c}$ is open in $O^{c}$ hence $\{X>\alpha\} \bigcup O$ is open in $\Omega$, this yields by Proposition 5.1

$$
\mathbb{C}(\{X>\alpha\}) \leq \mathbb{C}(\{X>\alpha\} \cup O)=\mathbb{C}_{\mathscr{P}}(\{X>\alpha\} \cup O) \leq \mathbb{C}_{\mathscr{P}}(\{X>\alpha\})+\varepsilon .
$$

Since $\varepsilon$ is arbitrary, the proof is complete thanks to Proposition 5.2.

Finally, we state an equivalent representation of $\mathbb{C}$ on $\mathcal{B}(\Omega)$.

Proposition 6. For any $A \in \mathcal{B}(\Omega), \mathbb{C}(A)=\inf \{\mathbb{C}(O) ; O \supset A, O$ open $\}$.

Proof. Let us consider $A \in \mathcal{B}(\Omega)$ and put $m=\inf \{\mathbb{C}(O) ; O \supset A, O$ open $\}$. Obviously, $m \geq \mathbb{C}(A)$. Let us prove the converse inequality: Let $\varepsilon>0$ and $\varphi \geq 1_{A}$ l.s.c. that we can always choose such that $0 \leq c_{\mathbb{E}}^{1}(\varphi)-\varepsilon \leq \mathbb{C}(A)$. Put $O=\{\varphi>1-\varepsilon\}$ which is an open set, then as $1_{O} \leq \frac{\varphi}{1-\varepsilon}$,

$$
m \leq \mathbb{C}(O) \leq c_{\mathbb{E}}^{1}\left(\frac{\varphi}{1-\varepsilon}\right)=\frac{c_{\mathbb{E}}^{1}(\varphi)}{1-\varepsilon} \leq \frac{\mathbb{C}(A)+\varepsilon}{1-\varepsilon} .
$$

As $\varepsilon$ is arbitrary small, this ends the proof.

\section{Appendix B. Proofs of the Theorems.}

B.1. Proof of Theorem 1. 1. $L\left(c_{\mathbb{E}}^{p}\right)=L^{p}(\mathscr{P})$ : The space $L\left(c_{\mathbb{E}}^{p}\right)$ from method 3 , departs from a different notion of negligible sets, based on the Lebesgue prolongation. To identify this space with $L^{p}(\mathscr{P})$ from method 1 , the same quotient space for the equivalence classes must be identified, see Proposition 5.4.

First of all, this $\mathbb{E}$ is regular as a consequence of Theorem 12 in [?]. Results of Section A.2 apply so that we have the representation

$$
\mathbb{E}[X]=\sup _{P \in \mathcal{M}^{1}(\mathbb{E})} E^{P}[X], X \in C_{b}(\Omega) .
$$

As a direct consequence of the last assertion of Theorem 5 , we have $\mathcal{M}^{1}(\mathbb{E})=\mathscr{P}$ and the representation (12) holds on $L\left(c_{\mathbb{E}}^{p}\right)$, we can apply all the results established in [?] and the space $L\left(c_{\mathbb{E}}^{p}\right)$ coincides with the space $L^{p}(\mathscr{P})$ (denoted by $\mathbb{L}_{\mathbb{C}_{\mathscr{P}}}^{p}$ in [?]). Moreover, $\mathbb{E}$ can be extended to any Borel function $X$ with $\sup _{P \in \mathscr{P}} E^{P}[|X|]<\infty$ via

$$
\mathbb{E}[X]=\sup _{P \in \mathscr{P}} E^{P}[X]
$$


2. $L\left(c_{\mathbb{E}}^{p}\right)=L^{p}(\mathbb{E})$ : As a consequence of Theorem 25 in [?], we have the desired result for any $p \in[1, \infty)$. The notions of quasi-continuity agree by Proposition 5 .

B.2. Proof of Theorem 2. By Proposition 4 any element in the dual space of $L\left(c_{\mathbb{E}}^{p}\right)$ is a Radon measure. Proposition 7 gives a representation of functionals in the dual of $L\left(c_{\mathbb{E}}^{p}\right)$. Theorem 2 then directly follows from Proposition 4 and 7 .

Proposition 7. Fix $p \in(1, \infty)$. A Radon measure $\mu$ on $(\Omega, \mathcal{B}(\Omega))$ which does not charge the $\mathbb{C}$-polar sets belongs to the dual space of $L\left(c_{\mathbb{E}}^{p}\right)$ if and only if there exists $P \in \mathscr{P}$ and $\psi \in L^{q}(P)$ such that $\mu=\psi \otimes P$ that is $\mu(\cdot)=\int . \psi(\omega) \mathrm{d} P(\omega)$.

More precisely $\mu$ belongs to the dual space $L\left(c_{\mathbb{E}}^{p}\right)^{*}$ if there is some $l \in L\left(c_{\mathbb{E}}^{p}\right)^{*}$ such that $l(X)=\int X \mathrm{~d} \mu$ for all $X \in L\left(c_{\mathbb{E}}^{p}\right)$. The case $p=1$ was considered in [?].

Proof. Case I: Assume first that $\mu$ is non-negative and that

$$
\left|\int_{\Omega} X(\omega) \mathrm{d} \mu(\omega)\right| \leq \mathbb{E}\left[|X|^{p}\right]^{1 / p}, \quad \forall X \in C_{b}(\Omega) .
$$

Define a set of measures $H=\left\{\mu(\cdot)=\int \cdot \psi \mathrm{d} P \mid P \in \mathscr{P}, \psi \in L^{q}(P), \psi \geq 0,\|\psi\|_{P, q} \leq 1\right\}$. It is easy to check that $H$ is a hereditary (i.e. $\mu \in H$ implies $\alpha \mu \in H \forall \alpha \in[0,1]$ ) convex set of measures. By the Prokhorov criterion, $H$ is relatively weakly compact.

Let us prove that $H$ is closed. Consider a sequence $\left(\mu_{n}\right)_{n}$ in $H$ converging weakly to the measure $\mu$. For all $n \in \mathbb{N}$, we have $\mu_{n}=\psi_{n} \otimes P_{n}$ where $P_{n} \in \mathscr{P}$ and $\psi_{n} \in L^{q}\left(P_{n}\right)$ with $\left\|\psi_{n}\right\|_{P_{n}, q} \leq 1$. As $\mathscr{P}$ is compact, by extracting a subsequence if necessary, we can assume that $\left(P_{n}\right)_{n}$ converges to an element $P$ weakly in $\mathscr{P}$. By the Hölder inequality for each $P_{n}$, we have for any $X \in C_{b}(\Omega)$ and for all $n \in \mathbb{N}$ :

$$
\left|\int X \mathrm{~d} \mu_{n}\right| \leq E^{P_{n}}\left[\left|\psi_{n} \| X\right|\right] \leq E^{P_{n}}\left[\left|\psi_{n}\right|^{q}\right]^{1 / q} E^{P_{n}}\left[|X|^{p}\right]^{1 / p} \leq\|X\|_{P_{n}, p} .
$$

Taking $n \rightarrow+\infty$, we get $\left|\int X \mathrm{~d} \mu\right| \leq E^{P}\left[|X|^{p}\right]^{1 / p}$, for all $X \in C_{b}(\Omega)$.

From this, it easily follows that there exists $\psi \in L^{q}(P), \psi \geq 0$ with $\|\psi\|_{P, q} \leq 1$ such that $\int X \mathrm{~d} \mu=E^{P}[\psi X]$ for all $X \in C_{b}(\Omega)$ which proves that $H$ is weakly compact.

Now, define the regular sublinear expectation by by

$$
\tilde{\mathbb{E}}[X]=\sup _{\mu \in H} \mu(X), \quad \forall X \in C_{b}(\Omega)
$$

with the notations introduced in the proof of Theorem 5, we have $H=\mathcal{M}(\tilde{\mathbb{E}})$. Now, using the fact that for each $P \in \mathcal{P}$, the dual space of $L^{p}(P)$ is $L^{q}(P)$ we get that if $X$ belongs to $C_{b}(\Omega)$ then $\mathbb{E}\left[|X|^{p}\right]^{1 / p}=\tilde{\mathbb{E}}[X]$. This implies that $\mu \in \mathcal{M}(\tilde{\mathbb{E}})=H$ and we have the desired result in this case.

Case II: Assume then that $\mu$ is positive. There exists $M \geq 0$ such that

$$
\left|\int_{\Omega} X(\omega) \mathrm{d} \mu(\omega)\right| \leq M \mathbb{E}\left[|X|^{p}\right]^{1 / p}, \quad \forall X \in C_{b}(\Omega) .
$$

By considering $\frac{\mu}{M}$ we obtain the result from case I with $\mu=\psi^{M} \otimes P, P \in \mathscr{P}$, and $\psi^{M}=M \cdot \psi \in L^{q}(P)$.

Case III: Finally, the general case is obtained by decomposing $\mu$ as $\mu^{+}-\mu^{-}$where $\mu^{+}$and $\mu^{-}$are singular and non-negative. From case II, we have

$$
\left|\int_{\Omega} X(\omega) \mathrm{d} \mu^{\circ}(\omega)\right| \leq M^{\circ} \cdot \mathbb{E}\left[|X|^{p}\right]^{1 / p}, \quad \forall X \in C_{b}(\Omega) \quad \circ \in\{+,-\} .
$$


Consequently $\frac{\mu^{+}}{M^{+}}$and $\frac{\mu^{-}}{M^{-}}$belongs to $H$, defined in Case I. Set $M=\max \left(M^{+}, M^{-}\right)$ and note $\frac{\mu^{+}}{M}, \frac{\mu^{-}}{M} \in H$. We have as in case II the following representations: $\frac{\mu^{+}}{M}=$ $\psi^{+} \otimes P^{+}, \frac{\mu^{-}}{M}=\psi^{-} \otimes P^{-}$, with $P^{+}, P^{-} \in \mathscr{P}, \psi^{+} \in L^{q}\left(P^{+}\right)$and $\psi^{-} \in L^{q}\left(P^{-}\right)$.

Now set $P=\frac{P^{+}+P^{-}}{2}$, we have $P \in \mathscr{P}$ by the convexity assumption on $\mathscr{P}$. Since

$$
P=\frac{1}{2}\left(P^{+}+P^{-}\right)=\frac{1}{2}\left(\psi^{+P}+\psi^{-P}\right) P, \quad \text { and } \psi^{+P}, \psi^{-P} \geq 0 \quad P-a . s .
$$

we have $\psi^{+P}, \psi^{-P} \leq 1 P$-a.s. With $\psi=M\left(\psi^{+} \psi^{+P}+\psi^{-} \psi^{-P}\right)$ we then have the desired representation $\mu=\psi \otimes P$. It remains to check:

$$
\|\psi\|_{P, q} \leq M\left\|\psi^{+} \psi^{+P}\right\|_{P, q}+M\left\|\psi^{-} \psi^{-P}\right\|_{P, q} \leq M\left(\left\|\psi^{+}\right\|_{P, q}+\left\|\psi^{-}\right\|_{P, q}\right)<\infty .
$$

This completes the proof.

\section{REFERENCES}

[1] C. Aliprantis And K. Border, Infinite dimensional analysis: a hitchhiker's guide, Springer Verlag, 2006.

[2] M. Avellaneda, A. Levy, and A. Paras, Pricing and hedging derivative securities in markets with uncertain volatilities, Applied Mathematical Finance, 2 (1995), pp. 73-88.

[3] P. Beissner, Coherent price systems and uncertainty-neutral valuation, Arxiv preprint arXiv:1202.6632, (2012).

[4] P. Beissner, Equilibrium prices and trade under ambiguous volatility, Economic Theory, (2016), pp. 1-26.

[5] N. BLOOM, The impact of uncertainty shocks, Econometrica, 77 (2009), pp. 623-685.

[6] B. Bouchard and M. Nutz, Arbitrage and duality in nondominated discrete-time models, The Annals of Applied Probability, 25 (2015), pp. 823-859.

[7] P. Carr and R. Lee, Volatility derivatives, Annu. Rev. Financ. Econ., 1 (2009), pp. 319-339.

[8] G. Choquet, Theory of capacities, in Annales de l'institut Fourier, vol. 5, Institut Fourier, 1954, pp. 131-295.

[9] R.-A. Dana and M. Jeanblanc, Financial markets in continuous time, Springer Science \& Business Media, 2007.

[10] C. Dellacherie, Analytical sets, capacities and haussdorff measures, lectures notes in mathematics, no. 180, 1972.

[11] L. Denis, M. Hu, And S. Peng, Function spaces and capacity related to a sublinear expectation: application to g-brownian motion paths, Potential analysis, 34 (2011), pp. 139-161.

[12] L. Denis AND M. Kervarec, Utility functions and optimal investment in non-dominated models, SIAM J. Control Optim., 51 (2013), pp. 1803-1822.

[13] L. Denis And C. Martini, A theoretical framework for the pricing of contingent claims in the presence of model uncertainty, The Annals of Applied Probability, 16 (2006), pp. 827-852.

[14] R. M. Dudley, Uniform central limit theorems, vol. 23, Cambridge Univ Press, 1999.

[15] L. G. Epstein And S. Ji, Ambiguous volatility and asset pricing in continuous time, Review of Financial Studies, 26 (2013), pp. 1740-1786.

[16] L. G. EPSTEIn AND S. JI, Ambiguous volatility, possibility and utility in continuous time, Journal of Mathematical Economics, 50 (2014), pp. 269-282.

[17] D. Feyel And A. De La Pradelle, Espaces de sobolev gaussiens, in Annales de l'institut Fourier, vol. 39, Institut Fourier, 1989, pp. 875-908.

[18] J.-P. Fouque AND B. REN, Approximation for option prices under uncertain volatility, SIAM Journal on Financial Mathematics, 5 (2014), pp. 360-383.

[19] I. Gilboa ANd D. SChmeidler, Maxmin expected utility with non-unique prior* 1, Journal of mathematical economics, 18 (1989), pp. 141-153.

[20] J. M. HARRISON AND D. M. KRePS, Speculative investor behavior in a stock market with heterogeneous expectations, The Quarterly Journal of Economics, (1978), pp. 323-336.

[21] J. M. HARRISON AND D. M. KRePs, Martingales and arbitrage in multiperiod securities markets, Journal of Economic theory, 20 (1979), pp. 381-408.

[22] M. Hu, F. WANG, AND G. Zheng, Quasi-continuous random variables and processes under the g-expectation framework, Stochastic Processes and their Applications, 126 (2016), pp. 2367-2387. 
[23] M. Kervarec, Etude des modéles non dominés en mathématiques financiéres, PhD thesis, Université d'Evry Val d'Essonne, France, (2008).

[24] F. KNight, Risk, uncertainty and profit, Beard Books, 1921.

[25] F. Maccheroni, M. Marinacci, and A. Rustichini, Dynamic variational preferences, Journal of Economic Theory, 128 (2006), pp. 4-44.

[26] A. Mas-Colell and W. Zame, Equilibrium theory in infinite dimensional spaces, Handbook of mathematical economics, 4 (1991), pp. 1835-1898.

[27] A. Matoussi, D. Possamaї, And C. Zhou, Robust utility maximization in nondominated models with 2bsde: the uncertain volatility model, Mathematical Finance, 25 (2015), pp. 258287.

[28] S. PENG, G-expectation, g-brownian motion and related stochastic calculus of itô type, Stochastic analysis and applications, The Abel Symposium 2005 (2006), pp. 541-567.

[29] S. Peng, Nonlinear expectations and stochastic calculus under uncertainty, Arxiv preprint ArXiv:1002.4546, (2010).

[30] J. Vorbrink, Financial markets with volatility uncertainty, Journal of Mathematical Economics, 53 (2014), pp. 64-78.

[31] J. Werner, Arbitrage and the existence of competitive equilibrium, Econometrica, (1987), pp. 1403-1418. 